\title{
Effect of CSF1R Inhibitor on Glial Cells Population and Remyelination in The Cuprizone Model
}

Fatemeh Tahmasebi ( $\sim$ fatemetahmasebi4@yahoo.com )

mashhad university https://orcid.org/0000-0002-0051-4648

\section{Shirin Barati}

Iran University of Medical Sciences

Iraj Ragerdi Kashani

Tehran University of Medical Sciences

\section{Original Article}

Keywords: Multiple sclerosis, PLX3397, Microglia, Oligodendrocyte, Astrocyte

Posted Date: February 8th, 2021

DOI: https://doi.org/10.21203/rs.3.rs-159459/v1

License: (c) (1) This work is licensed under a Creative Commons Attribution 4.0 International License. Read Full License 


\section{Abstract}

Multiple sclerosis is a kind of autoimmune and demyelinating disease and its pathological symptoms include inflammation, myelin loss, astrocytosis, and microgliosis. The colony stimulating factor 1 receptor (CSF1R), as an essential factor for the microglial function, and PLX3397 is its specific inhibitor. In this study, we assessed the effect of different doses of PLX3397 for microglial ablation on glial cells population and remyelination process. Sixty male C57BL/ 6 mice ( 8 weeks old) were divided into 6 groups. The animals were fed with $0.2 \%$ cuprizone diet for 12 weeks for chronic demyelination model induction. For microglial ablation, PLX3397 $(290 \mathrm{mg} / \mathrm{kg})$ was added to the animal food for 3, 7, 14 and 21 days. Glial cells number was measured using immunohistochemistry. We evaluated the rate of remyelination using electron microscopy and Luxol Fast Blue staining. The expression levels of all genes were assessed by qRT-PCR method. Data were analysed using Graph pad prism and SPSS software.Data showed that the administration of different doses of PLX3397 significantly (pष0.001) reduced microglial cells. PLX3397 administration significantly ( $\mathrm{p} \otimes 0.001)$ increased oligodendrocytes population with along rise of the remyelination compared to the cuprizone mice, which was confirmed by the results of LFB and TEM. Gene results showed that PLX3397 treatment reduced CSF1R expression. According to results, the administration of PLX3397 for 21 days enhanced remyelination by increasing oligodendrocytes in the chronic demyelination model. These positive effects could be related to the reduction of microglia.

\section{Introduction}

Multiple sclerosis (MS) is a kind of autoimmune disease characterized with multifocal lesions in the central nervous system (CNS), which affect almost 2.5 million people worldwide (Goldmann and Prinz 2013). It is a chronic, neurodegenerative disorder and its pathological symptoms consist of inflammation, myelin damage and axonal injury, astrocytosis, and microgliosis (Kramann et al. 2016).

Microglia, as CNS resident innate immune cells, respond to inflammation by the secretion of inflammatory cytokines that exacerbate disease symptoms. As such, they play a vital role in both healthy and pathological conditions (Nimmerjahn, Kirchhoff, and Helmchen 2005). The activated microglia observed in injured regions of the CNS in the cuprizone model contribute to the inflammatory process (Gudi et al. 2014). Some studies suggest that axonal degeneration in diseases such as MS is a result of inflammation and chronic demyelination (Bjartmar et al. 2000; Dutta et al. 2006).

When CNS homeostasis changes, the microglia are activated and generally distributed throughout the CNS close to neurons, astrocytes and oligodendrocytes (Szepesi et al. 2018). The extreme activation of glial cells damages CNS via signaling molecules such as inflammatory cytokines, reactive oxygen/nitrogen species and glutamate in neurodegenerative diseases (Thameem Dheen, Kaur, and Ling 2007). The recent research reveals that an microglial inflammatory response is related to the bioactive and inflammatory factors secretion (Suzuki et al. 2004), which further promotes neuroinflammation and neurodegeneration (Monif, Burnstock, and Williams 2010). 
Microglia can destruct oligodendrocytes and the myelin sheath through antigens presentation and proinflammatory molecules secretion (Prinz and Priller 2014). Several reports have reported wide damage and the apoptosis of mature oligodendrocytes (Wolswijk 2000) with oxidized DNA and lipids in these cells along with myelin loss in MS diseases (Bizzozero et al. 2005). Remyelination is the myelin repair procedure following demyelination. Viability and proliferation of oligodendrocytes are the key to the remyelination process, followed by proliferation, recruitment and differentiation of oligodendrocyte precursor cells (OPCs) into mature myelinating oligodendrocytes (Domingues et al. 2016).

In the cuprizone model, astrogliosis and microgliosis accompany demyelination at damaged regions (Petković et al. 2016). In response to the CNS injury or inflammation, astrocytes and microglial cells play beneficial and destructive role, respectively (Bihler et al. 2017). Astrocytes, as another population of glial cells, modulate neuroinflammation but are necessary for the support of neurons and the preservation of the blood-brain barrier (Jäkel and Dimou 2017). The beneficial or detrimental effects of microglia and astrocytes on MS progression are unclear (Correale and Farez 2015). The microglial cells development is related to Irf8 and the colony stimulating factor 1 receptor (CSF1R) (Gibson, Geraghty, and Monje 2018). As an essential marker for microglial cells function, CSF1R has two ligands, IL-34 and CSF1 (Patel and Player 2009). CSF1 adjusts viability, proliferation and differentiation of microglia, and reduced densities of macrophages in several tissues was seen in mice lacking either CSF1R or CSF1 (Elmore et al. 2014b). It has been shown that under normal brain conditions, microglia are the only special cell types that express the CSF1R (Elmore et al. 2014a).

Pexidartinib, or PLX3397, as a small selective CSF1R inhibitor molecule, crosses the blood-brain barrier and targets the CSF1/CSF1R pathway. It quickly ablates microglial cells in the CNS. PLX3397 can interact with the CSF1R juxtamembrane area (Giustini et al. 2018). Therefore, PLX3397 administration can deplete microglia through CSF1R inhibition. In this study, we investigated the role of microglial ablation on demyelination process and number of glial cells using different doses of PLX3397 as CSF1R inhibitor in the cuprizone model. We assessed the cellular and molecular mechanisms of interaction between microglia and astrocytes/oligodendrocytes, demonstrating that different doses of PLX3397 can decrease demyelination via microglial reduction.

\section{Materials And Methods}

\subsection{Animals}

In the present study, sixty male mice C57BL/6 (8 weeks) were obtained from Pasteur Institute, Iran. They were preserved for 1 week in the Animal Breeding Centre at Tehran University of Medical Sciences under normal conditions to adapt to the environment before the study. All experimental procedures were in compliance with ethical laws of Tehran University of Medical Sciences.

\subsection{Experimental Groups}


The mice were divided into six groups $(\mathrm{n}=10)$ including: Control (Ctrl) group fed by normal food; Cuprizone (CPZ) group fed with a mixed diet containing 0.2\% CPZ (Sigma-Aldrich, St. Louis, MO) for 12 weeks in order to induce the chronic demyelination model of MS. After 12 weeks of cuprizone administration, PLX3397 (Active Biochem, Hong Kong), a CSF1R inhibitor for microglial depletion, was given to mice as the mixed diet. The PLX3, PLX7, PLX14 and PLX21 groups were treated with the PLX3397 (dose of $290 \mathrm{mg} / \mathrm{kg}$ ) for 3, 7, 14 and 21 days, respectively. At the end of this study, animals were first deeply anesthetized with ketamine/xylazine (ratio 10:1), and then transcardially perfused with phosphate-buffered saline (PBS) (Sigma-Aldrich, St. Louis, MO). For histological procedures, mice were perfused with $4 \%$ paraformaldehyde (PFA) (Sigma-Aldrich, St. Louis, MO, USA). We used $2.5 \%$ glutaraldehyde and 4\% PFA perfusion for electron microscopy studies. For the molecular level analysis, after perfusion with saline, corpus callosum (CC) tissues were immediately dissected. Samples were rapidly transferred to liquid nitrogen tanks and then froze and kept at $-80^{\circ} \mathrm{C}$.

\subsection{Luxol Fast Blue (LFB) Staining}

The LFB staining was used for the evaluation of myelination processes in white matters on paraffin sections. The myelinated fibres were blue and demyelinated areas were white in this staining. After preparing 5 - $\mu \mathrm{m}$ paraffin sections of the brain, tissue processing was performed. Deparaffinised sections were incubated in LFB solution $(0.01 \%)$ overnight at $60^{\circ} \mathrm{C}$. Excess dye was washed off by distilled water. Then, after air drying sections, they were covered with DPX mounting medium. The LFB stained sections were assessed under a light microscope (Olympus, Japan) and images were captured by a camera. In the end, the percentage of myelinated zone was calculated in six experimental groups.

\subsection{Immunohistochemistry}

Firstly, brains were fixated with 4\% PFA, and they were embedded in paraffin after dehydration. Coronal sections of brain tissues were cut by a microtome (Microm, Walldorf, Germany) for antigen retrieval, and immersed into citrate buffer before being permeabilized by $20 \%$ tween for $30 \mathrm{~min}$. For nonspecific markers blocking, we used $0.1 \% \mathrm{BSA}$ in $0.1 \%$ triton for $1 \mathrm{~h}$. Then, sections were incubated at $4^{\circ} \mathrm{C}$ with primary antibodies: anti-Iba-1 (1:250; Wako chemicals, Virginia), anti-GFAP (1:100; Gene Tex, CA) and anti-Olig2 (1:100; Gene Tex, CA) overnight. After the addition of secondary antibodies (Vector Labs, CA), slides were washed with PBS twice; the nuclei were stained with DAPI (4', 6-diamidino-2-phenylindole) for $1 \mathrm{~min}$. Finally, slides were assessed by a fluorescent microscope (Olympus BX51TRF, Tokyo, Japan) equipped with a digital camera (Olympus) for capturing images.

\subsection{Real Time-PCR}

For gene evaluation, the total messenger RNA (mRNA) of mice CC in experimental groups was extracted by the Trizol (Invitrogen, CA), chloroform and isopropanol, respectively, and then washed with $75 \%$ ethanol. We used nearly $5 \mu \mathrm{g}$ of mRNA for complementary DNA (cDNA) synthetize. Afterwards, the cDNA synthesis was performed with cDNA synthesis kit (Fermentase, Lithuania) for all samples and almost 100 ng of cDNA was enhanced by Power SYBR Green Master mix (Applied Biosystems, California, USA). 
All samples were evaluated in triplicate and analysed with the StepOne Software (Thermo Fisher Scientific, Waltham, MA). The gene expression was compared to GAPDH (Glyceraldehyde 3-phosphate dehydrogenase) as the reference gene. The data were analysed by the $\triangle \mathrm{CT}$ method and the results were normalized relative to the control, and a value of 1 was obtained. In this study, primer sequences for qRTPCR were summarized in Table 1.

Table 1: Primer sequences for each gene using the qRT-PCR.

\begin{tabular}{|c|c|c|}
\hline Genes & Forvard Sequence $\left(5^{\circ}\right.$ to 3$)$ & Reverse Sequence ( 5 to 3 ) \\
\hline CSFIR & AGTGTCCTTAGAGGTGGTTTC & TGAAAGGGAACAGAGGAAGAG \\
\hline GFAP & GTGGAGAGGGACAACTTTGC & TTCATCTGCCTCCTGTCTATACG \\
\hline Olig2 & GAACCCCGAAAGGTGTGGAT & TTCCGAATGTGAATTAGATTIGAGG \\
\hline TNF- $\alpha$ & TGTAGCCCACGTCGTAGCAA & AGGTACAACCCATCGGCTGG \\
\hline IL-6 & GGTACATCCTCGACGGCATCT & GT GCCTCTTTGCTGCTTTCAC \\
\hline GAPDH & CAAGCTCATTTCCTGGTATGACAA & GGGATAGGGCCTCTCTTGCT \\
\hline
\end{tabular}

\subsection{Transmission Electron Microscopy (TEM)}

In this study, to assess ultrastructures of myelin and axons in experimental groups, we used TEM images. After brain fixation with $2.5 \%$ glutaraldehyde for $2 \mathrm{~h}$ and the dissection of $\mathrm{CC}$, the tissues were washed three times with PBS, and then fixed by $1 \%$ osmicacid, before triple washing by PBS. Consequently, after samples dehydration, they were embedded in resin. For the ultrathin section preparation, we performed ultramicrotome before using uranyl acetate and lead citrate. Finally, sections were assessed by a CM120 EM followed by assessment and photographing of sections by TEM (LEO 906; Carl-Zeiss, Jena, Germany).

\subsection{Rotarod Test}

In this study, the rotarod test was used for the analysis of motor coordination and balance. This test was performed by an accelerating rotarod (IITC Life Science Inc, CA). To do so, mice were placed on the rotating drum, and this process was repeated three times a day for 3 consecutive days. Firstly, each mouse was placed on the drum for 3 min with progressive acceleration from 4 to $35 \mathrm{rpm}$. $24 \mathrm{~h}$ after the test, mice were evaluated on the drum with the same speed for $5 \mathrm{~min}$. The time required for each mouse to maintain its balance on the drum was recorded as latency to fall.

\subsection{Data analysis}

The results of present study were analysed by the Statistical Package for the Social Sciences software (SPSS, ver. 24). The differences between groups were assessed by one-way analysis of variance (ANOVA) 
followed by the Tukey test. Also, GraphPad Prism program for chart drawing (Version 7). Data were expressed as the mean \pm SEM. The significance is considered by $p \leq 0.05$.

\section{Results}

\subsection{The results of QRT-PCR}

The specific genes of glial cells including microglia (CSF1R), astrocytes (GFAP) and oligodendrocytes (Olig2) in the CC of animals were evaluated by real time PCR method. The qRT-PCR data revealed that the chronic cuprizone-induced model enhanced the expression level of CSF1R and GFAP genes in comparison with the Ctrl group ( $\mathrm{p} \leq .001$ ), which was confirmed by microgliosis and astrocytosis in the corpus callosum region in this model (Fig. 1A, B). Moreover, the mRNA levels revealed that the administration of PLX3397, as the CSF1R inhibitor, with varying periods such as 3, 7, 14 and 21 days significantly ( $p \leq .001)$ reduced the gene expression of CSF1R compared to the cuprizone group. The PLX3397 treatment for 21 days was more effective in terms of microglial ablation, so that more than $95 \%$ of the microglia were removed after 21 days (Fig. 1A). However, the PLX3397 administration for various days did not have a significant effect on the expression level of GFAP gene compared to CPZ mice (Fig. 1B). Besides, the data indicated that oligodendrocytes rate dropped in the cuprizone group ( $\mathrm{p} \leq .001 \mathrm{vs}$. control), suggesting myelin destruction in this model. Furthermore, the results revealed that PLX3397 treatment for 7, 14 and 21 days could significantly increase Olig2 gene level compared to CPZ mice (Fig. 1C). PLX3397 treatment for 21 days with the more than $95 \%$ microglial ablation had the highest effect on increasing oligodendrocyte number.

The results of this part demonstrated that PLX3397 administration could inhibit CSF1R receptor as a specific marker of microglia and decrease these cells in the cuprizone model without affecting astrocytosis. However, PLX3397 treatment increased the population of oligodendrocytes after the cuprizone demyelination model.

To discover how microglial ablation in the corpus callosum of CPZ mice responds to immune factors, we assessed the inflammatory factors by the qRT-PCR. The results suggested that astrocytosis and microgliosis in the cuprizon model could increase ( $\mathrm{p} \leq .001 \mathrm{vs}$. control) the expression level TNF- $a$ and IL6 as pro-inflammatory factors (Fig. 2A, B). The analysed data showed that the depletion of microglia with PLX3397 for 3, 7, 14 and 21 days leads to dramatic reductions in the expression of pro-inflammatory genes, including TNF-a and IL-6 (Fig. 2A, B). PLX3397 treatment for 21 days with greater microglial ablation had the highest effect on the reduction of inflammatory factors. Also, microglial elimination can inhibit inflammation by decreasing pro-inflammatory cytokines including IL-6 and TNF-a.

\subsection{The results of immunofluorescence}

In the present study, we assessed the effects CSF1R inhibitor, PLX3397, on glial population in the CPZ mice using the immunofluorescence (IF) method. Firstly, the microglial number was calculated at the gene level using the real time PCR in CC with CSF1R genes (as the target of PLX3397) following PLX3397 
administration. Also, these gene results of PLX3397 treatment was confirmed by IF of Iba-1 (green) as a specific marker of microglia in the CC (Fig. 3A). As shown in IF images, microgliosis in the CPZ group and PLX administration could reduce microglial number (Fig. 3A). The quantitative analysis of data exhibited that cuprizone administration significantly $(p \leq 0.001)$ increased the number of Iba-1 possitive cells compared to the Ctrl (Fig. 3B). The PLX treatment for $3,7,14$ and 21 days significantly ( $\leq 0.001$ ) reduced microglial population in comparison with CPZ group (Fig. 3B). Hence, after 21 days, the microglial number dropped to more than $95 \%$ compared to these cells in the cuprizone model.

These results showed microgliosis in the CPZ model and PLX3397, as a CSF1R inhibitor, can be used for the reduction and depletion of microglia.

After the real time qRT-PCR assessment, astrocytes number was measured at the protein level by the IF of GFAP, and astrogliosis was observed in the CC images of cuprizone mice (Fig. 4A). The quantitative analysis of data showed that $\mathrm{GFAP}^{+}$cells significantly rose in the $\mathrm{CPZ}$ group compared to the Ctrl $(\mathrm{p} \leq .001)$ and the expression of GFAP protein was not significantly different in the PLX treatment groups after 3, 7, 14 and 21 days compared to the CPZ group (Fig. 4B). Results of the qRT-PCR and IF indicated that cuprizone administration led to astrogliosis after 12 weeks and PLX3397, as CSF1R inhibitor, had no effect on astrogliosis in the CPZ model.

After oligodendrocyte evaluation at the gene expression level, we also assessed these cells with the Olig2 protein marker using IF technique in six experimental groups. The IF images displayed a reduction in the expression of Olig2 marker, indicating a drop in oligodendrocytes number in the cuprizone group (Fig. $5 A)$. The quantitative analysis of the immunofluorescence sections exhibited that the number of Olig2 positive cells decreased significantly ( $\mathrm{p} \leq .001)$ in CPZ vs. Ctrl. Also, PLX3397 treatment for 3 and 7 days had no significant effect on oligodendrocytes number, but PLX3397 administration for 14,21 days upregulated the expression of the Olig2 (Fig 5B). These results indicate oligodendrocyte reduction, which is a sign of demyelination and impossibility of spontaneous remyelination in the corpus callosum in the cuprizone model. PLX3397 administration for 14 and 21 days increased oligodendrocyte number in this model.

\subsection{LFB results for the assessment of myelin damage recovery}

In this study, we used LFB staining to evaluate demyelination and remyelination levels in six groups. The myelination part in LFB-stained CC sections was in blue in the Ctrl group. However, the demyelination region was in white in the $\mathrm{CPZ}$ group, which indicated cuprizone addition to the mice diet for 12 weeks for the induction of chronic demyelination in CC (Fig. 6A). Also, the quantitative analysis displayed that PLX $(290 \mathrm{mg} / \mathrm{kg}$ ) administration for 3 and 7 days had no significant effect on remyelinayion process. However, PLX (290 mg/kg) treatment for 14 and 21 days significantly increased remyelination rate in CC following demyelination induction by the cuprizone (Fig. 6B).

\subsection{TEM results for the assessment of myelin damage recovery}


In this study, cuprizone digestion for 12 weeks damaged myelin sheath and axons. Therefore, we used TEM for assessing the ultrastructure of myelin sheaths and axons in CPZ group and groups received PLX3397 for 3, 7, 14 and 21 days. The TEM images showed that in the CPZ group, as opposed to other groups, the number of demyelinated fibres increased. Also, myelin sheaths disruption with a gap between myelin layers as well as axons destruction were observed (Fig. 7). The electron microscopy images display that PLX treatment for 3 and 7 days has no effect on the recovery of myelin and axonal damage. However, PLX administration for 14 and 21 days could bridge the gap between myelin sheaths layers and reduce the number of demyelinated fibres (Fig. 7), which were consistent with LFB images.

\subsection{A rotarod test for the assessment of motor deficits}

The rotarod test is extensively used to assess the motor coordination of rodents. It is especially sensitive to detecting motor dysfunctions. In the present study, we used this test to evaluate balance and motor coordination in cuprizone-induced demyelination model treated with different doses of PLX3397. The results showed that cuprizone administration for 12 weeks significantly $(p \leq .001)$ reduced mobility time on the rotarod system compared to the Ctrl group. This suggests that chronic cuprizone model can induce motor deficits. The PLX treatment for 3 and 7 days had no effect on mean running time, but mice receiving PLX for 14 and 21 days stayed longer on the drum related to the CPZ group (Fig. 8). The results illustrate that PLX administration for 14 and 21 days can improve motor deficit via microglial ablation in the cuprizone model.

\section{Discussion}

The current therapies for neurodegenerative diseases such as MS are effective only in a minority of patients. Also, these treatments can only slow disease progression but they cannot deter disease process and lead to remyelination and repair. For this reason, comprehensive studies of factors involved in the pathogenesis of MS are needed to recognize novel therapeutic objectives and create suitable therapies for MS patients. MS is a chronic inflammatory autoimmune demyelinating disease of the CNS that is characterized with oligodendrocyte loss, astrogliosis and microgliosis (Liu et al. 2017). It has been shown that microglia activation in MS can exacerbate this disease (Nelson, Soma, and Lavi 2002). Knowing that the activated microglia-dependent inflammation is associated with the pathogenesis of MS, we focused on the effect of PLX3397 administration for 3, 7, 14 and 21 days as a specific CSF1R inhibitor for microglial ablation on remyelination and glial cells population in the cuprizone demyelination model.

The cuprizone model is a toxic experimental model (without an autoimmune component) with pathogenesis comparable to MS (Kipp et al. 2009). In this study, we first used the chronic cuprizone model, after which microglia were ablated by the CSF1R inhibitor (PLX3397) according to our previous study (Tahmasebi et al. 2020). Cuprizone-induced demyelination model in animals is used to study MSrelated lesions. It is characterized by the degeneration of oligodendrocytes and the demyelination of diverse brain regions that consist of the corpus callosum (Kipp et al. 2009). For chronic demyelination induction, we used cuprizone diet for 12 weeks. Moreover, the cuprizone model is similar to III and IV MS 
types in humans. The chronic cuprizone model leads to oligodendrocyte apoptosis, astrogliosis, demyelination, microglia cell activation, and phagocytosis of myelin sheaths (Kipp et al. 2009).

Microglia, as CNS resident innate immune cells, are in a quiescent state with thin processes under physiological conditions; however, they are enlarged with retracted processes under the pathological stimulation (Monif, Burnstock, and Williams 2010). The researches showed that microglia expressed NADPH oxidase and increased reactive oxygen specious on the one hand and produced inflammatory factors and contributed to axonal injury and neurodegeneration on the other hand (van Horssen et al. 2012). Therefore, microglial ablation can slow disease progression, provide neuroprotection and increase myelin and axonal repair by reducing harmful pro-inflammatory factors and oxidative stress (Airas, Nylund, and Rissanen 2018). It has been shown that CSF1R signalling is necessary for the survival and proliferation of microglia. PLX3397 administration can ablate microglia via the CSF1R inhibition. Numerous studies have demonstrated that inhibiting microglial activation attenuates the severity of EAE (Popovic et al. 2002).

In this study, we observed astrogliosis and microgliosis in IF images and real time PCR analysis of the cuprizone model. In line with our results, Andrew et al., (2012) reported that cuprizone caused microgliosis and astrogliosis in the corpus callosum (Steelman, Thompson, and Li 2012). Moreover, the results of IF and qRT- PCR showed that PLX3397 administration for 3, 7, 14 and 21 days lead to microglial ablation in the cuprizone model. The data suggest that PLX treatment for 21 days decreased to microglial number to more than $95 \%$ compared to the cells in the cuprizone model, but different doses of PLX administration had no effect on astrocytes population, which is similar to the study of Tahmasebi et al. (2020) (Tahmasebi et al. 2020).

The activated microglia release various inflammatory chemokines that aggravate the disease severity (Nelson, Soma, and Lavi 2002). The qRT-PCR results showed that IL-6 and TNF-a were upregulated in the cuprizone group and PLX3397 administration in different days downregulated these pro-inflammatory factors. In keeping with our study, Wang et al. (2019) revealed that activated microglia produced TNF-a in EAE mice, which could induce extreme glutamate and lead to the dysfunction of neurons and oligodendrocytes (Wang et al. 2019).

Remyelination is the procedure of myelin formation and repair that ensues demyelination. Remyelination usually fails in chronic MS due to disarrayed differentiation of OPCs. In the present study, the results of IF and qRT-PCR revealed that Olig2 marker level in corpus callosum decreased in the cuprizone group, representing oligodendrocyte population downregulation and demyelination. According to our study, Kuhlmann et al. demonstrated that microglia activation caused oligodendrocyte apoptosis, both of which are two histopathological hallmarks of the cuprizone animal model (Kuhlmann et al. 2008). However, PLX3397 administration with microglial ablation for 14 and 21 days could increase oligodenerocyte number in the cuprizon model. Haider et al. reported the DNA and lipid oxidation of oligodendrocytes and neurons, together with inflammation, could cause demyelination, axonal and neuronal damage in MS (Haider et al. 2011). 
The recent data shows that activated microglia, as a major source of oxidative stress, release ROS and NO radicals and play a crucial role in neurodegenerative disease (Fischer et al. 2013). OPCs are at risk of oxidative stress due to higher levels of proapoptotic proteins and lower levels of antiapoptotic proteins and antioxidant enzymes (Butts, Houde, and Mehmet 2008).

The demyelination and remyelination rate in six experimental groups were evaluated by the LFB staining. Our results revealed that the induction of chronic cuprizone model decreased myelination by more than $90 \%$, and PLX3397 treatment for 3 and 7 days had no effect on remyelination. However, PLX3397 administration for 14 and 21 days, reducing activated microglia and pro-inflammatory factors, enhanced remyelination and increased oligodenerocyte number. In the same vein, Slowik et al. (2015) observed the corpus callosum demyelination of C57BL/ $6 \mathrm{~J}$ mice during $0.2 \%$ cuprizone treatment using by LFB-stained sections (Slowik et al. 2015).

Finally, the ultrastructures of myelin sheath and axon were analysed by TEM. The results indicated that healthy myelin structure was disrupted, and there was a gap between myelin sheath layers in CPZ mice. Also, the number of demyelinated fibres increased after cuprizone digestion. PLX3397 administration for 3 and 7 days had no effect on remyelination while PLX3397 therapy for 14 and 21 days led to myelin repair, as verified by LFB results. In addition, Cruz-Martinez et al. (2016) studied the effect of intraventricular injection of mesenchymal stem cells on remyelination in a chronic demyelinating model. The electron microscopy images in their study showed that the chronic cuprizone model impaired myelin sheath and increased demyelinated axons, as verified by the TEM results (Cruz-Martinez et al. 2016).

In sum, we found that PLX3397 administration for 14 and 21 days after cuprizon model induction can enhance remyelination and axonal repair through microglial ablation, pro-inflammatory factors reduction and increased oligodendrocyte number.

\section{Conclusion}

Our study demonstrated that cuprizone model, as one of the MS models, can give rise to demyelination with oligodendrocytes loss, microgliosis and astrocytosis. Also, we revealed that different doses of PLX3397 administration ablated microglial cells in the brains of CPZ mice. Microglial depletion significantly suppressed microglial activation and decreased neuroinflammation and neurological deficits. Thus, microglial cells play a vital role in the pathogenesis of the MS disease and ablation of these cells can offer a suitable approach for the treatment of MS patients.

\section{Declarations}

\section{Author contribution statement}

Fatemeh Tahmasebi carried out the experiment and wrote the manuscript. Iraj Ragerdi Kashani conceived the original idea and supervised the project. Shirin Barati performed the analytic calculations. All authors discussed the results and contributed to the final manuscript. 
Availability of data and material

Not applicable

Compliance with ethical standards

Funding

This study was supported by a grant (number 95.04.3033645) received from the Tehran University of Medical Sciences and Health Services, Tehran, Iran.

\section{Ethical approval}

All experimental procedures were carried out in accordance with the guidelines of the Ethic Committee of Tehran University of Medical Science (TUMS), Tehran, Iran. All efforts were made to reduce the number of animals used and their suffering.

\section{Conflict of Interests}

The authors had no conflict of interest regarding the publication of this paper.

\section{Consent for publication}

Not applicable. There aren't any patient in this research study.

\section{Consent to participate}

Not applicable. There aren't any patient in this research study.

\section{Acknowledgment}

This study was supported by Tehran University of Medical Sciences and Health Services, Tehran, Iran.

\section{References}

1. Airas L, Nylund M, Eero Rissanen (2018) 'Evaluation of microglial activation in multiple sclerosis patients using positron emission tomography', Front Neurol, 9: 181

2. Bihler K, Kress E, Esser S, Nyamoya S, Tauber SC, Clarner T, Stope MB, Pufe T, Lars-Ove B (2017) 'Formyl peptide receptor 1-mediated glial cell activation in a mouse model of cuprizone-induced demyelination'. J Mol Neurosci 62:232-243

3. Bizzozero OA, Gisela DeJesus K, Callahan, and Andrzej Pastuszyn (2005) 'Elevated protein carbonylation in the brain white matter and gray matter of patients with multiple sclerosis'. J Neurosci Res 81:687-695 
4. Bjartmar, Carl G, Kidd S, Mörk R, Rudick, Bruce DT (2000) 'Neurological disability correlates with spinal cord axonal loss and reduced $\mathrm{N}$-acetyl aspartate in chronic multiple sclerosis patients'. Ann Neurol 48:893-901

5. Butts BD, Houde C, and H Mehmet (2008) 'Maturation-dependent sensitivity of oligodendrocyte lineage cells to apoptosis: implications for normal development and disease'. Cell death differentiation 15:1178

6. Correale J, Mauricio F, Farez (2015) 'The role of astrocytes in multiple sclerosis progression'. Front Neurol 6:180

7. Cruz-Martinez, Pablo, Susana González-Granero, Maira Micaela Molina-Navarro, Jesus PachecoTorres, Jose Manuel Garcia-Verdugo, Emilio Geijo-Barrientos, Jonathan Jones, and Salvador Martinez. 2016. 'Intraventricular injections of mesenchymal stem cells activate endogenous functional remyelination in a chronic demyelinating murine model', Cell death \& disease, 7: e2223

8. Domingues HS, Camila C, Portugal R, Socodato, João B, Relvas (2016) 'Oligodendrocyte, astrocyte, and microglia crosstalk in myelin development, damage, and repair'. Frontiers in cell developmental biology $4: 71$

9. Dutta R, McDonough J, Yin X, Peterson J, Chang A, Torres T, Gudz T, Macklin WB, Lewis DA, Robert JF (2006) 'Mitochondrial dysfunction as a cause of axonal degeneration in multiple sclerosis patients'. Ann Neurol 59:478-489

10. Elmore MR, Pittman AR, Najafi MA, Koike, Nabil Nazih Dagher, Elizabeth Erin Spangenberg, Rachel Anne Rice, Masashi Kitazawa, Bernice Matusow, Hoa Nguyen, and Brian Lee West (2014a) 'CSF1 receptor signaling is necessary for microglia viability, which unmasks a cell that rapidly repopulates the microglia-depleted adult brain', Neuron, 82: 380

11. Elmore MRP, Allison R, Najafi, Maya A, Koike, Nabil N, Dagher EE, Spangenberg RA, Rice M, Kitazawa B, Matusow H, Nguyen, Brian LW (2014b) 'Colony-stimulating factor 1 receptor signaling is necessary for microglia viability, unmasking a microglia progenitor cell in the adult brain'. Neuron 82:380-397

12. Fischer M, Therese I, Wimmer R, Höftberger S, Gerlach L, Haider T, Zrzavy S, Hametner D, Mahad, Christoph J, Binder, and Markus Krumbholz. 2013. 'Disease-specific molecular events in cortical multiple sclerosis lesions', Brain, 136: 1799-815

13. Gibson EM, Anna C, Geraghty, Monje M (2018) 'Bad wrap: Myelin and myelin plasticity in health and disease', Developmental neurobiology, 78: 123-35

14. Giustini N, Bernthal NM, Bukata SV, Arun SS (2018) 'Tenosynovial giant cell tumor: case report of a patient effectively treated with pexidartinib (PLX3397) and review of the literature'. Clinical sarcoma research 8:14

15. Goldmann T, and Marco Prinz (2013) 'Role of microglia in CNS autoimmunity', Clinical and Developmental Immunology, 2013

16. Gudi V, Gingele S, Skripuletz T, Stangel M (2014) 'Glial response during cuprizone-induced de-and remyelination in the CNS: lessons learned'. Frontiers in cellular neuroscience 8:73 
17. Haider L, Fischer MT, Frischer JM, Bauer J, Höftberger R, Botond G, Esterbauer H, Christoph J, Binder JL, Witztum, Hans Lassmann (2011) 'Oxidative damage in multiple sclerosis lesions', Brain, 134: 1914-1924

18. Jäkel S, and Leda Dimou (2017) 'Glial cells and their function in the adult brain: a journey through the history of their ablation'. Frontiers in cellular neuroscience 11:24

19. Kipp M, Clarner T, Dang J, Copray S, and Cordian Beyer (2009) 'The cuprizone animal model: new insights into an old story', Acta neuropathologica, 118: 723-36

20. Kramann N, Menken L, Hayardeny L, Hanisch U-K, Wolfgang Brück (2016) 'Laquinimod prevents cuprizone-induced demyelination independent of Toll-like receptor signaling', NeurologyNeuroimmunology Neuroinflammation, 3: e233

21. Kuhlmann T, Miron V, Cuo Q, Wegner C, Antel J, and W Brück (2008) 'Differentiation block of oligodendroglial progenitor cells as a cause for remyelination failure in chronic multiple sclerosis', Brain, 131: 1749-58

22. Liu Y, Given KS, Harlow DE, Matschulat AM, Wendy B, Macklin JL, Bennett, Gregory PO (2017) 'Myelin-specific multiple sclerosis antibodies cause complement-dependent oligodendrocyte loss and demyelination'. Acta neuropathologica communications 5:1-13

23. Monif M, Burnstock G, David AW (2010) 'Microglia: proliferation and activation driven by the P2X7 receptor'. Int J Biochem Cell Biol 42:1753-1756

24. Nelson, Peter T, Lorinda A, Soma, Lavi E (2002) 'Microglia in diseases of the central nervous system'. Annals of medicine 34:491-500

25. Nimmerjahn A, Kirchhoff F, Helmchen F (2005) 'Resting microglial cells are highly dynamic surveillants of brain parenchyma in vivo'. Science 308:1314-1318

26. Patel S, Mark RP (2009) 'Colony-stimulating factor-1 receptor inhibitors for the treatment of cancer and inflammatory disease'. Current topics in medicinal chemistry 9:599-610

27. Petković F, Campbell IL, Gonzalez B, Bernardo Castellano (2016) 'Astrocyte-targeted production of interleukin- 6 reduces astroglial and microglial activation in the cuprizone demyelination model: Implications for myelin clearance and oligodendrocyte maturation', Glia, 64: 2104-2119

28. Popovic N, Schubart A, Goetz BD, Zhang S-C, Linington C, lan DD (2002) 'Inhibition of autoimmune encephalomyelitis by a tetracycline'. Annals of Neurology: Official Journal of the American Neurological Association the Child Neurology Society 51:215-223

29. Prinz M, and Josef Priller (2014) 'Microglia and brain macrophages in the molecular age: from origin to neuropsychiatric disease'. Nat Rev Neurosci 15:300-312

30. Slowik A, Schmidt T, Beyer C, Amor S, Clarner T, Kipp M (2015) 'The sphingosine 1-phosphate receptor agonist FTY 720 is neuroprotective after cuprizone-induced CNS demyelination'. Br J Pharmacol 172:80-92

31. Steelman AJ, Jeffrey P, Thompson, Li J (2012) 'Demyelination and remyelination in anatomically distinct regions of the corpus callosum following cuprizone intoxication'. Neuroscience research $72: 32-42$ 
32. Suzuki T, Hide I, Ido K, Kohsaka S, Inoue K, Yoshihiro Nakata (2004) 'Production and release of neuroprotective tumor necrosis factor by P2X7 receptor-activated microglia', J Neurosci, 24: 1-7

33. Szepesi Z, Manouchehrian O, Bachiller S, and Tomas Deierborg. 2018. 'Bidirectional microglianeuron communication in health and disease', Frontiers in cellular neuroscience, 12

34. Tahmasebi F, Pasbakhsh P, Barati S, Madadi S, Iraj RK (2020) 'The effect of microglial ablation and mesenchymal stem cell transplantation on a cuprizone-induced demyelination model', Journal of Cellular Physiology

35. Thameem Dheen S, Charanjit Kaur, Eng-Ang Ling (2007) 'Microglial activation and its implications in the brain diseases'. Curr Med Chem 14:1189-1197

36. van Horssen J, Singh S, van der Pol S, Kipp M, Lim JL, Peferoen L, Gerritsen W, Kooi E-J, Witte ME, Jeroen JGG (2012) 'Clusters of activated microglia in normal-appearing white matter show signs of innate immune activation'. J Neuroinflamm 9:156

37. Wang J, Wang J, Wang J, Yang B, Weng Q, He Q (2019) 'Targeting microglia and macrophages: a potential treatment strategy for multiple sclerosis'. Front Pharmacol 10:286

38. Wolswijk G (2000) 'Oligodendrocyte survival, loss and birth in lesions of chronic-stage multiple sclerosis', Brain, 123: 105-15

\section{Figures}
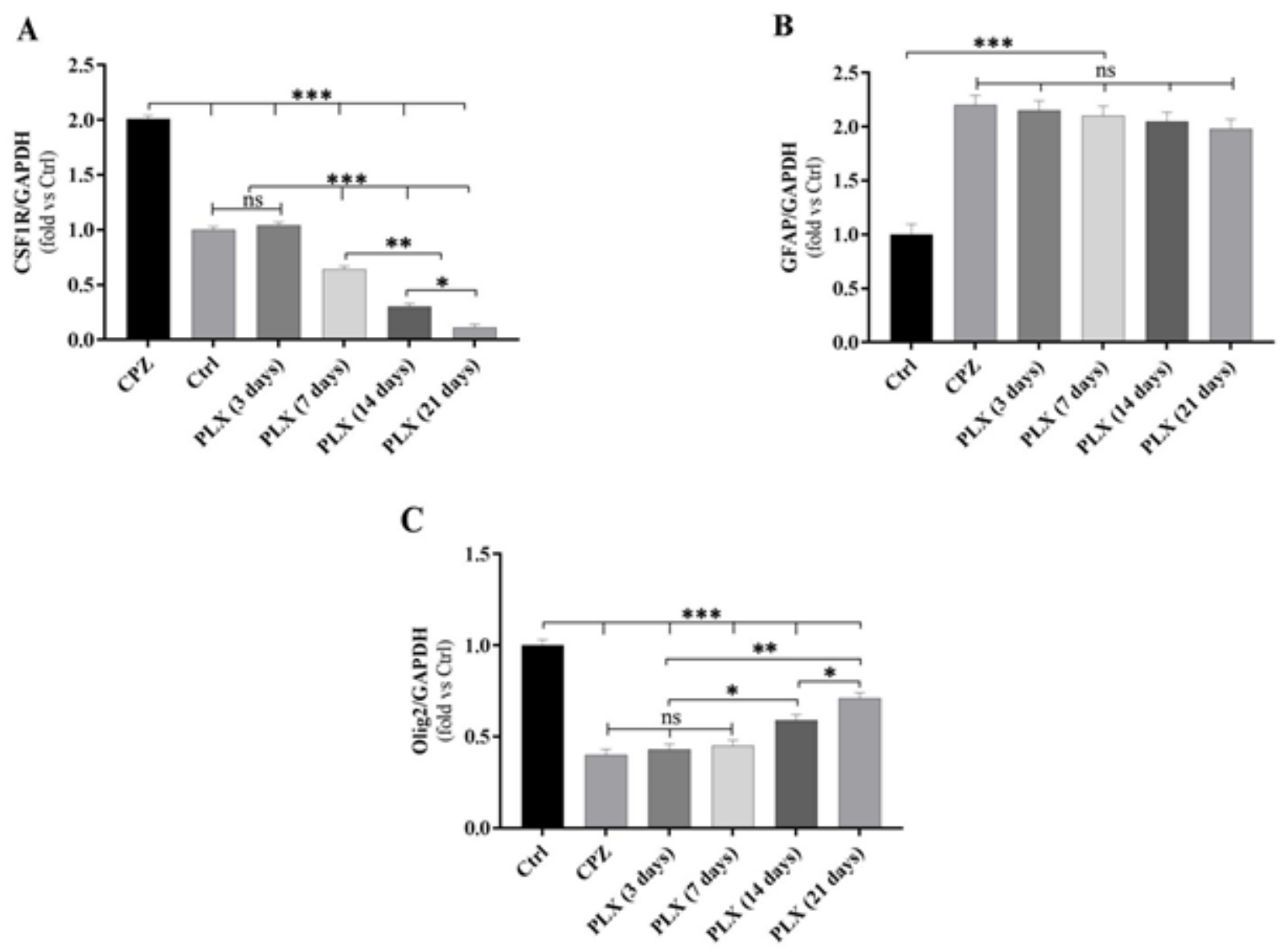


\section{Figure 1}

The effect of different doses of PLX3397 administration (3, 7, 14 and 21) on genes of glial cells assessed by qRT-PCR in the cuprizone model. The real-time PCR using specific primer for (A) CSF1R as a specific gene for microglia, (B) GFAP for astrocytes and (C) Olig2 as a oligodendrocyte gene. The data analysis manifested the rising expressions of CSF1R and GFAP with a reduction in Olig2 level in the untreated cuprizone group; however, PLX3397 digestion reduces microglial specific gene without affecting astrocytes genes. The expression levels of Olig2 gene increased in PLX3397 treatment after 7, 14 and 21 days. GAPDH was as housekeeping gene for normalization of gene expression. Values are expressed as the mean \pm SEM. ${ }^{* \star *} \mathrm{p} \leq .001,{ }^{*} \mathrm{p} \leq .01$ and ${ }^{*} \mathrm{p} \leq .05$. CPZ, cuprizone; $\mathrm{qRT}-\mathrm{PCR}$, quantitative real time polymerase chain reaction; SEM, standard error of mean.
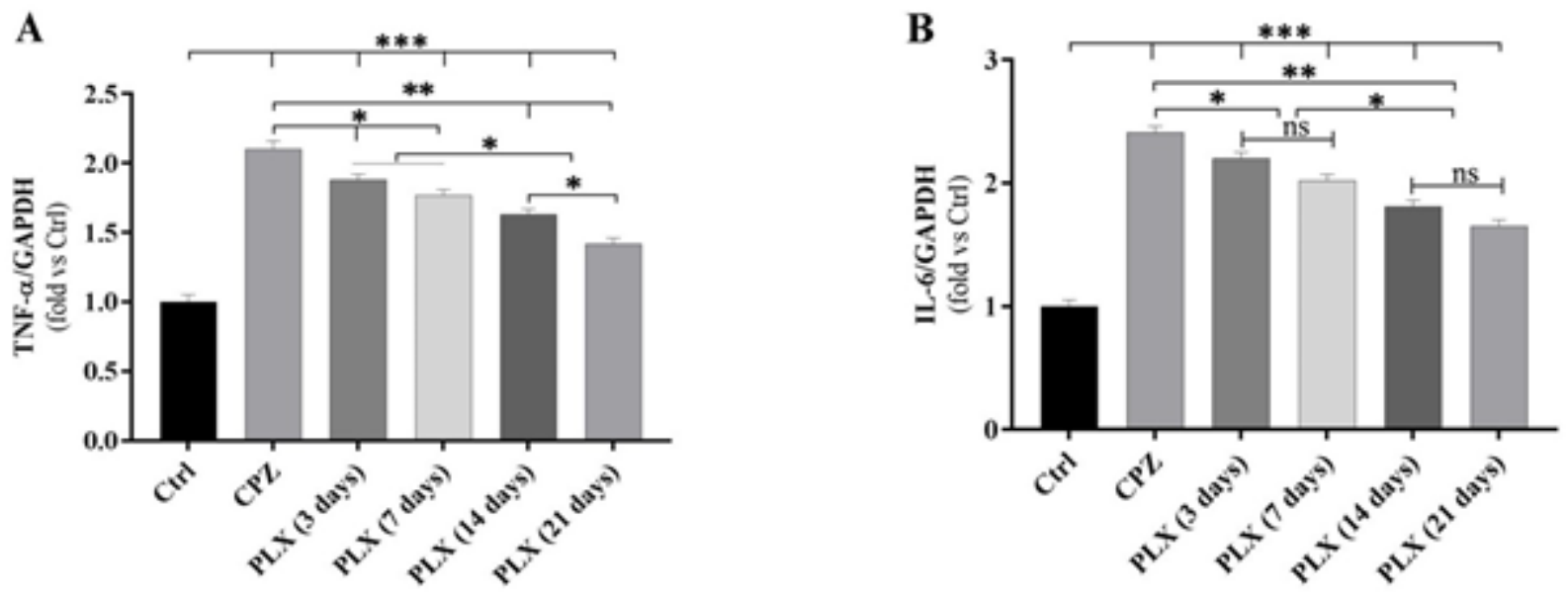

\section{Figure 2}

The effect of PLX3397 administration for 3, 7, 14 and 21 days on pro-inflammatory genes in the cuprizone model were assessed by qRT-PCR. (A, B) The relative mRNA expression of TNF- $a$ and IL-6 revealed that expression levels of TNF- $\alpha$ and IL- 6 genes soared after CPZ treatment vs. Ctrl. The PLX3397 treatment for varying days decreased inflammatory genes in the CPZ model. GAPDH was a housekeeping gene for the normalization of gene expression. Values are expressed as the mean \pm SEM. ${ }^{\star \star \star} p \leq .001$, ${ }^{* *} \mathrm{p} \leq .01$ and ${ }^{*} \mathrm{p} \leq .05$. CPZ, cuprizone; qRT-PCR, quantitative real time polymerase chain reaction; SEM, standard error of mean. 


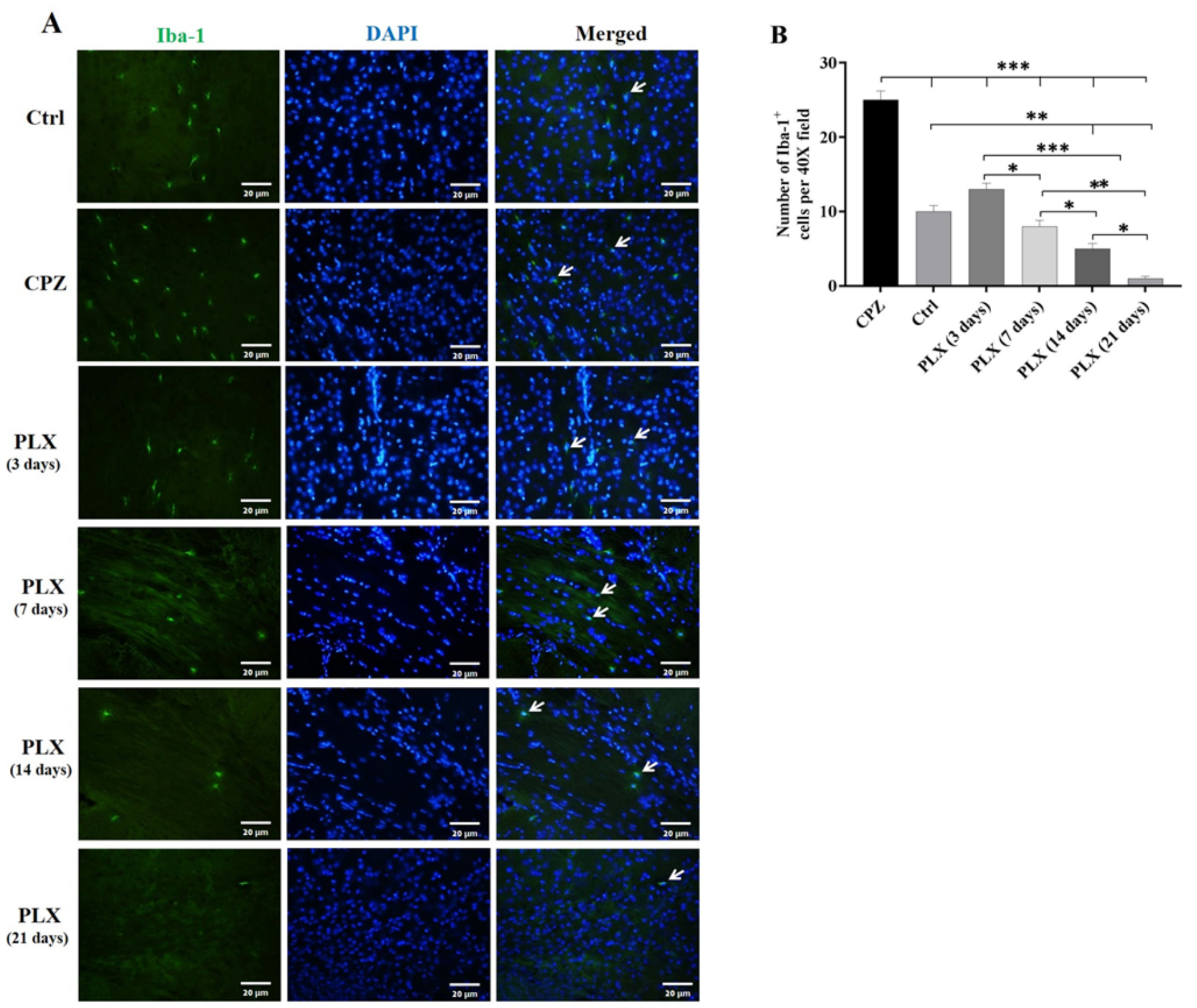

\section{Figure 3}

IF assessment of microglial cell specific marker. (A) These images show the $\mathrm{CC}$ sections of microglial cell (Iba-1, green) and nuclei (DAPI, blue). Arrows point to the nuclei overlap with Iba-1 positive cells (40x field). (B) Quantification of IF images revealed augmented Iba-1 level in the CPZ group, and PLX therapy significantly diminished this marker. Values are expressed as the mean $\pm S E M$. ${ }^{* \star} p \leq .001$, ${ }^{\star \star} p \leq .01$ and ${ }^{*} \mathrm{p} \leq$.05. CC, corpus callosum; CPZ, cuprizone; IF, immunofluorescence; DAPI, 4',6-diamidino-2phenylindole. 
A

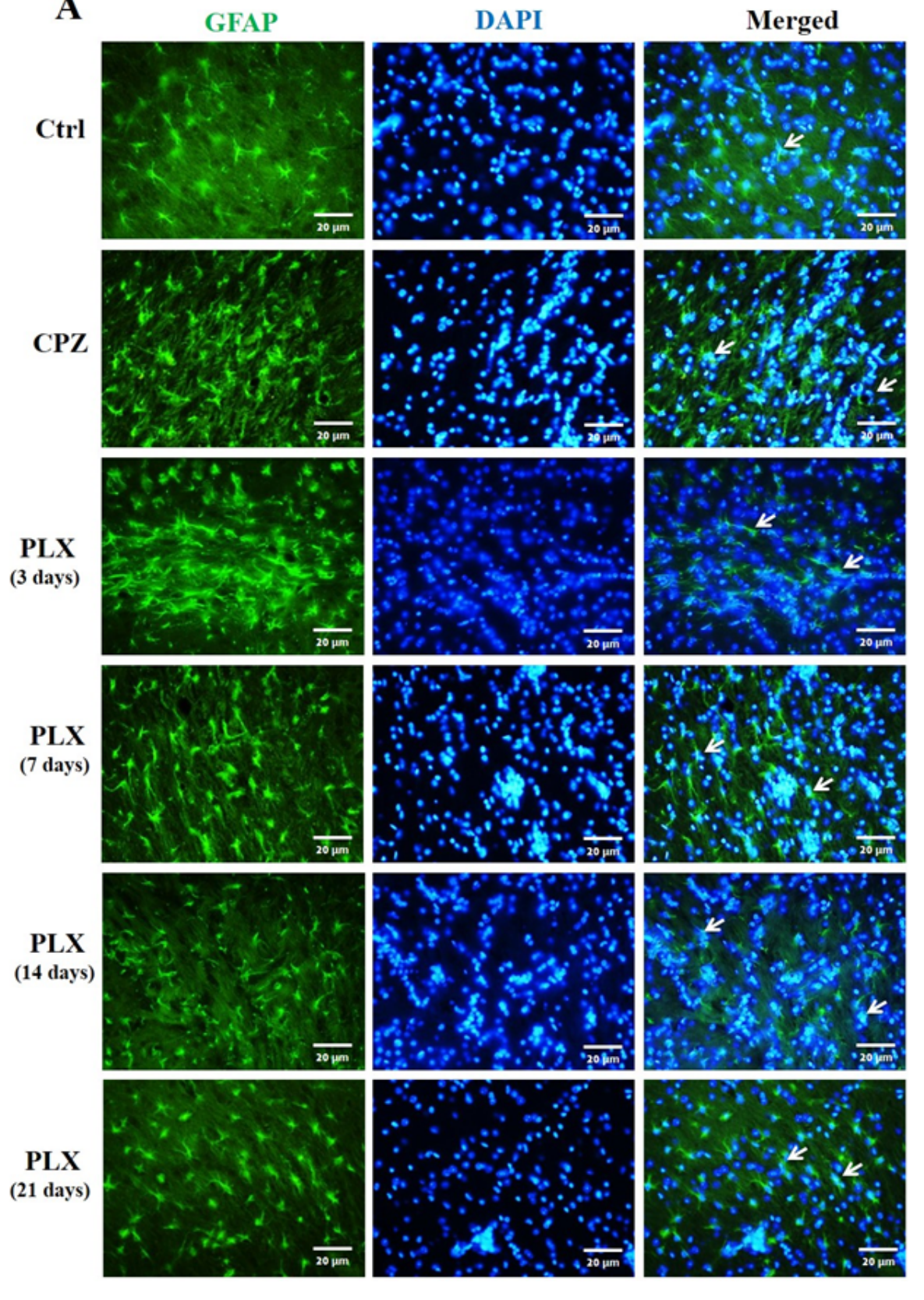

B

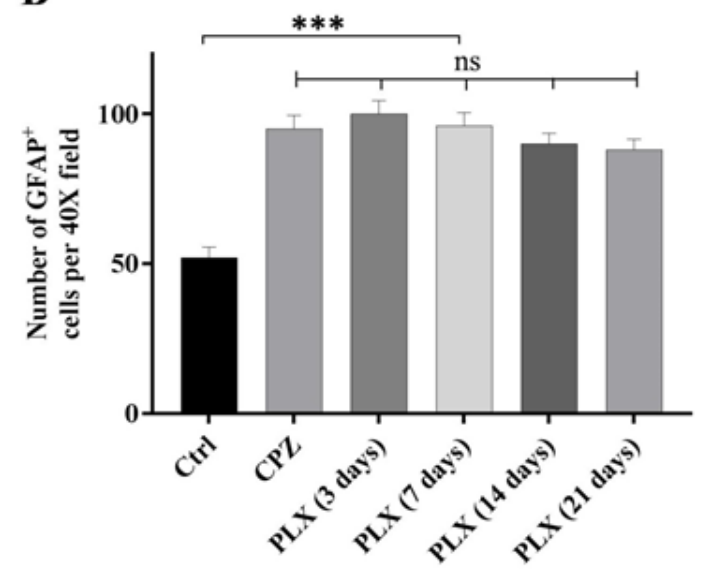

Figure 4

The evaluation of astrocyte specific marker with IF. (A) These images exhibit CC sections of astrocytes (GFAP, green) and nuclei (DAPI, blue). Arrows point to nuclei overlap with GFAP positive cells (40x field). (B) Quantification of IF images displayed an increase in GFAP+ of CPZ mice and PLX3397 was unable to significantly reduce this protein expression. Values are expressed as the mean $\pm \mathrm{SEM}$ and ${ }^{\star \star *} \mathrm{p} \leq .001$. CC, corpus callosum; CPZ, cuprizone; IF, immunofluorescence; DAPI, 4',6-diamidino-2-phenylindole. 


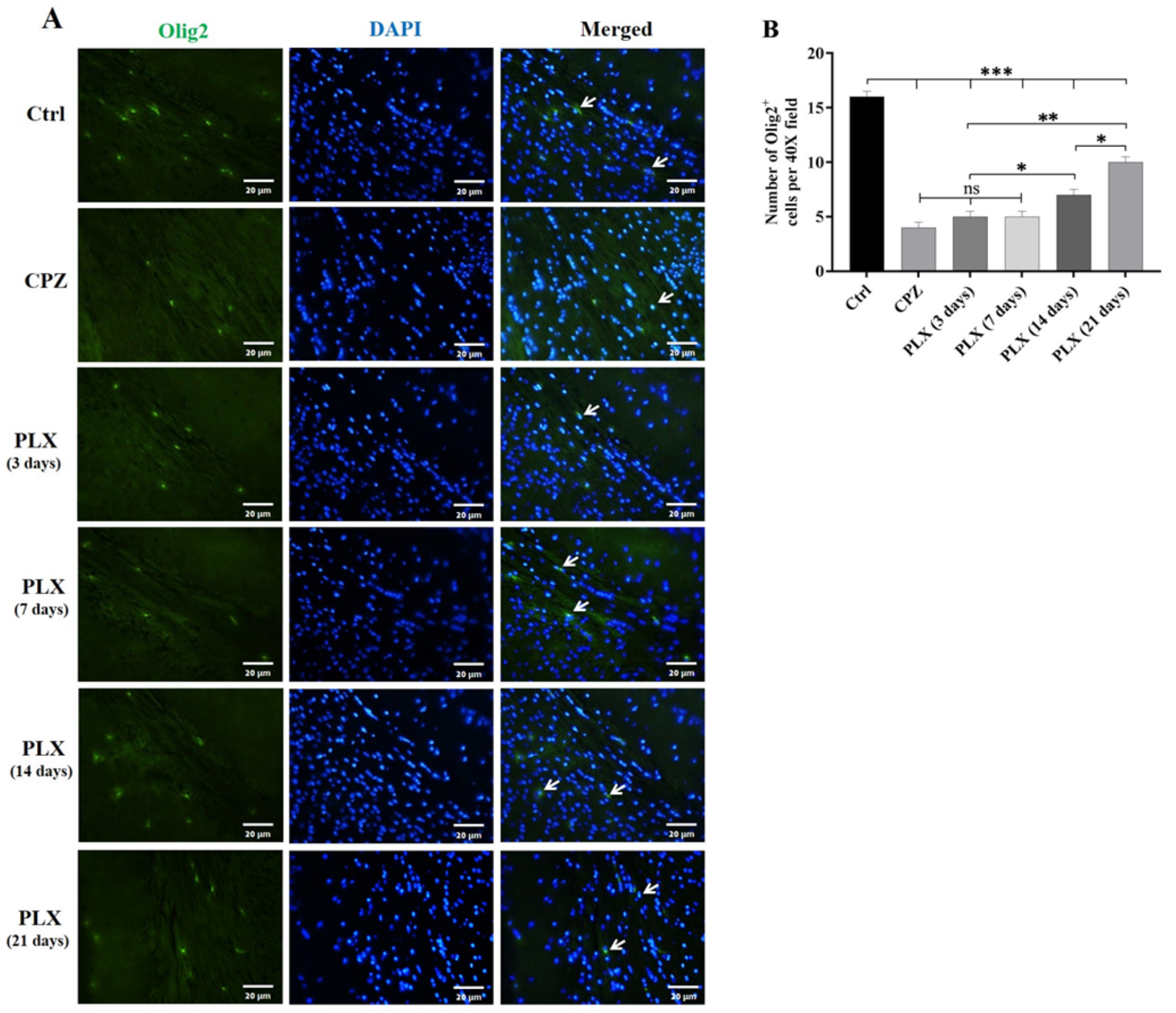

Figure 5

Immunofluorescence evaluation of oligodendrocyte specific marker. (A) These IF images are CC sections of oligodendrocytes (Olig2, green) and nuclei (DAPI, blue). Arrows point to nuclei overlap with Olig2 positive cells in merged pictures (40x field). (B) The quantitative analysis of IF images displayed downregulation in Olig2 protein in the CPZ mice, and PLX administration could significantly increase this marker after 14, 21 days. Values are expressed as the mean \pm SEM. ${ }^{* *} p \leq .001,{ }^{*} p \leq .01$ and ${ }^{*} p \leq .05$. CC, corpus callosum; CPZ, cuprizone; DAPI, 4',6-diamidino-2-phenylindole; IF, immunofluorescence. 
A

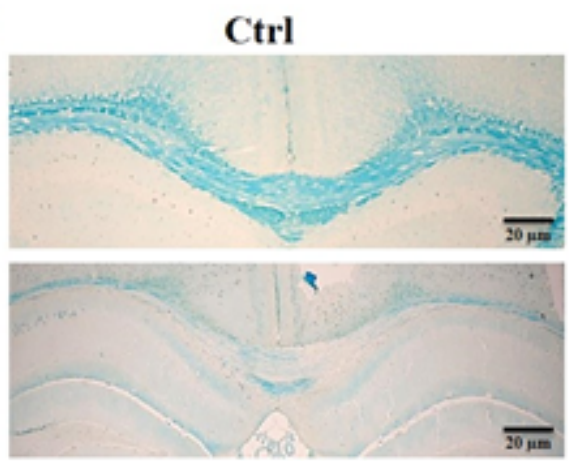

PLX (7 days)
CPZ
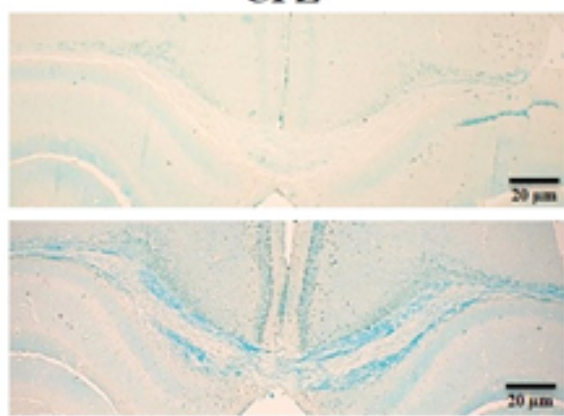

PLX (14 days)
PLX (3 days)
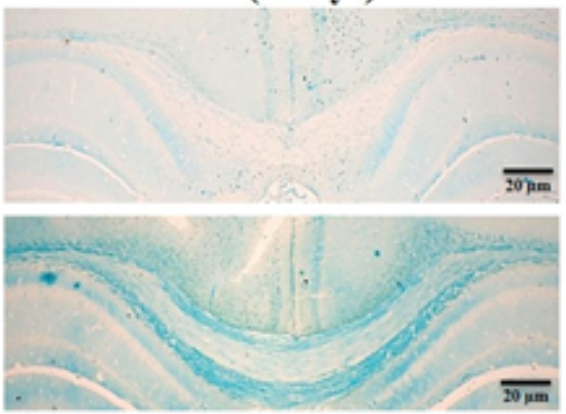

PLX (21 days)

B

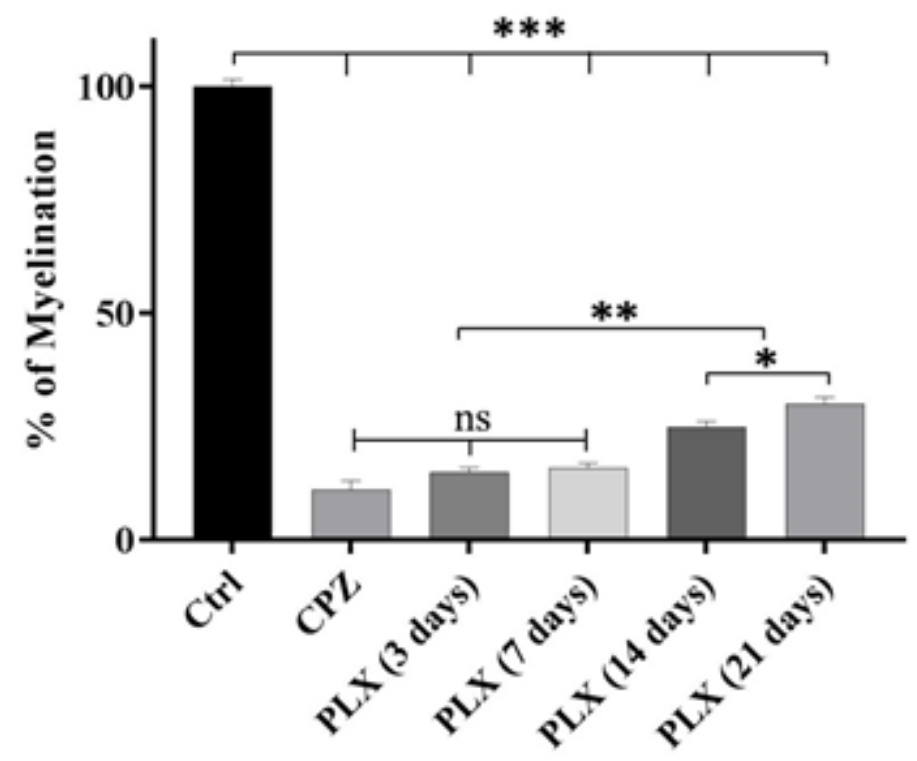

Figure 6

Effect of PLX3397 administration for 3, 7, 14 and 21 days on myelin damage recovery in corpus callosum. (A) LFB staining was used for myelin sheath evaluation. (B) Quantitative analysis of LFBstained sections of CC indicated an increase in the demyelinated zone in the CPZ mice, which declined after PLX treatment for 14 and 21 days. Values are expressed as mean \pm SEM . ${ }^{\star \star \star} p \leq .001,{ }^{\star \star} p \leq .01$ and ${ }^{*} \mathrm{p} \leq .05$. CC, corpus callosum; CPZ, cuprizone; LFB, Luxol Fast Blue. 


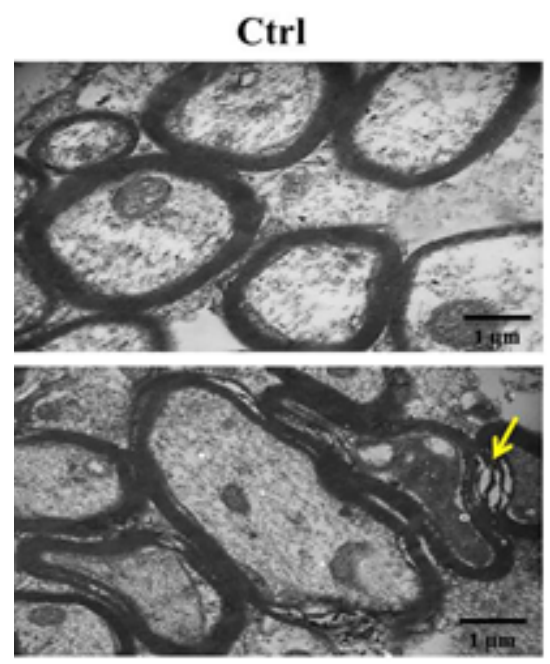

PLX (7 days)

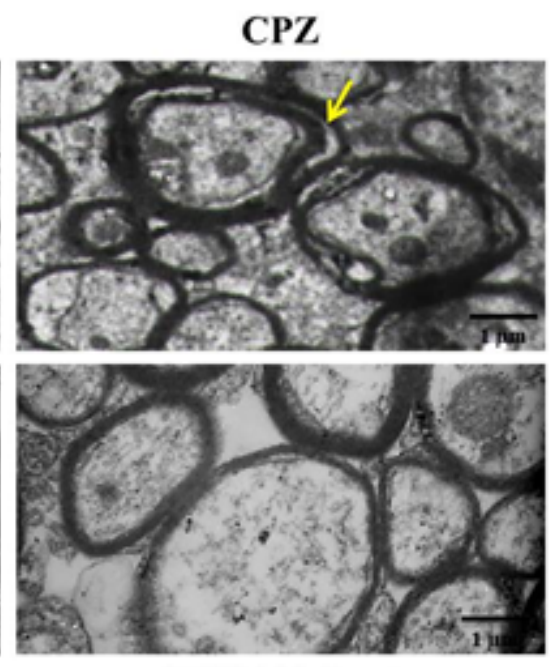

PLX (14 days)
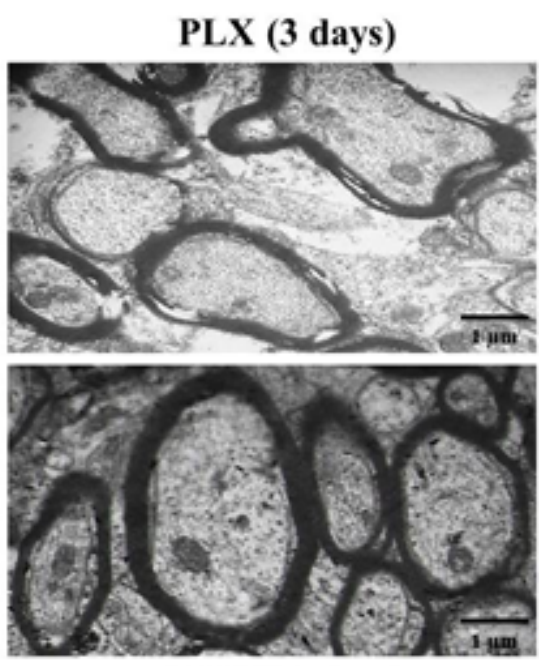

PLX (21 days)

\section{Figure 7}

Assessment of PLX3397 treatment on ultrastructures of axon and myelin sheaths in the cuprizone model using TEM images. In this study, we observed disruptions and gaps between layers of myelin sheaths in the cuprizone mice. TEM sections revealed that PLX3397 could diminish myelin deficits in the cuprizone mice after 14 and 21-day administration. The yellow arrow points to damaged myelin sheath. CPZ, cuprizone; TEM: Transmission electron microscopy.

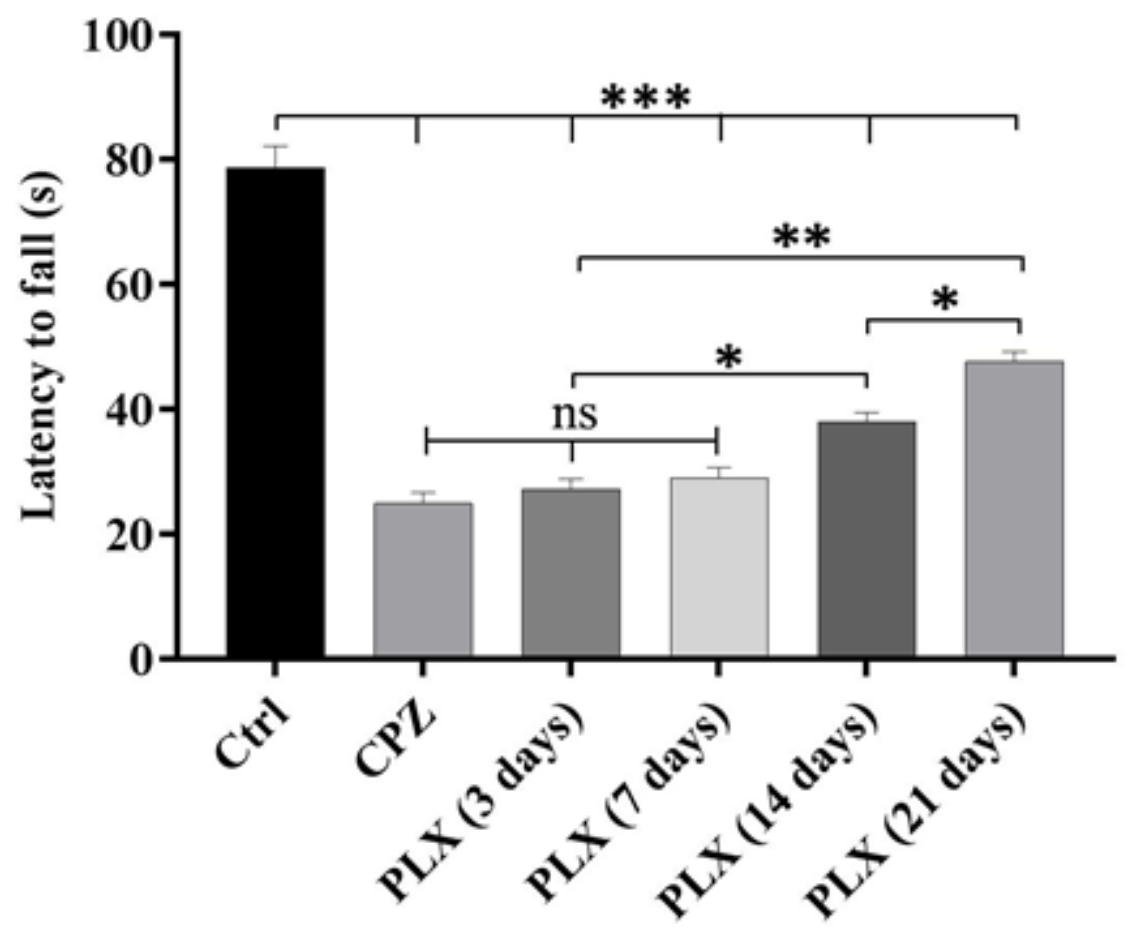

Figure 8 
Assessments of the effect of PLX3397 treatment for 3, 7, 14 and 21 days on neurological behavioural deficits in cuprizone mice. The results of rotarod assay showed that cuprizone digestion diminished motor coordination, while PLX3397 administration improved neurological behavioural deficits. Values are expressed as mean \pm SEM. ${ }^{* \star *} \mathrm{p} \leq .001,{ }^{*} \mathrm{p} \leq .01$ and ${ }^{*} \mathrm{p} \leq .05$. CPZ, cuprizone; $\mathrm{SEM}$, standard mean error. 